\title{
Stochasticity among Victims of COVID-19 Pandemic
}

\author{
Ramalingam Shanmugam (iD) \\ Gerald Ledlow ${ }^{2}$ \\ Karan P Singh iD $^{3}$ \\ 'School of Health Administration, Texas \\ State University, San Marcos, TX, 78666 \\ USA; ${ }^{2}$ Department of Healthcare Policy, \\ Economics and Management, School of \\ Community and Rural Health, The \\ University of Texas at Tyler Health \\ Science Center, Tyler, TX, I1937, USA; \\ ${ }^{3}$ Department of Epidemiology and \\ Biostatistics, The University of Texas at \\ Tyler Health Science Center, Tyler, TX, \\ I1937, USA
}

\begin{abstract}
This article provides a thorough explanation of methods and theoretical concepts to detect infectivity of COVID-19. The concept of heterogeneity is discussed and its impacts on COVID-19 pandemics are explored. Observable heterogeneity is distinguished from nonobservable heterogeneity. The data support the concepts of heterogeneity and the methods to extract and interpret the data evidence for the conclusions in this paper. Heterogeneity among the vulnerable to COVID-19 is a significant factor in the contagion of COVID-19, as demonstrated with incidence rates using data of a Diamond Princess cruise ship. Given the nature of the pandemic, its heterogeneity with different social norms, pre- and post-voyage quick testing procedures ought to become the new standard for cruise ship passengers and crew. With quick testing, identification of those infected and thus, not allowing to embark on a cruise or quarantine those disembarking, and other mitigation strategies, the popular cruise adventure could become norm for safe voyage. The novel method used in this article adds valuable insight in the modeling of disease and specifically, the COVID-19 virus.
\end{abstract}

Keywords: observed heterogeneity, non-observed heterogeneity, over dispersion, under dispersion, Poisson distribution, binomial distribution, Tango's test statistics

\section{Introduction}

In the literature, the term heterogeneity echoes differently in one context versus another. Its root word lies in Greek "heterogenes" meaning different. In scientific disciplines, the word heterogeneity is popularly mentioned to have existed when the variance is large. In insurance applications, for example, the premium is assessed more if an insurer is in a heterogeneous group with high hazard proneness. ${ }^{5}$ The large (small) variance is indicative of heterogeneity (homogeneity). Ecochard ${ }^{6}$ has interesting discussions for heterogeneity. In healthcare disciplines, heterogeneity is referred to as different outcomes among patients. The utilized mathematical expressions are eased in this section with added descriptions. The main topic is all about detecting reasons for complex infectivity of COVID-19 and they squarely connect to their heterogeneity. There are two types of heterogeneity. One is the observable heterogeneity, and the other is non-observable heterogeneity. We have devised a novel method to identify and estimate the level of each type in this article. The heterogeneity is linked with a nonobservable hidden trait as done in genetics. The heterogeneity refers dissimilar attributes across the subgroups of the population itself even before sampling. In a sense, the heterogeneity really points to the non-identical nature in a random sample or population. Sometimes, the heterogeneity implies a shifting stochasticity. In genetic studies, several authors refer to genetic heterogeneity as rather too difficult to ascertain. They really mean that because the alleles in more than one locus exhibit susceptibility to any disease including COVID-19, there is a need to track the loci
Correspondence: Karan P Singh

Email karan.singh@uthct.edu 
to infer their heterogeneity. So, in a sense, the application of heterogeneity is really a discussion of an opposite to similarity across loci. The reader is referred to Elston et al (2002, pages 3404-344) for details. ${ }^{2}$ Hope and Norris ${ }^{7}$ attempted to determine how heterogeneity played a role in judgements in the context of crime victimization. A formal definition of heterogeneity is examined later in the article and its properties are explored and itemized.

We use the notations $y, \theta$, and $H_{0}$ to describe the observation, the unknown parameter, and the posed conjecture (in other words, hypothesis) pertinent to the chance-oriented mechanism behind the COVID-19 pandemic. However, in the literature, using a random sample $y_{1}, y_{2}, \ldots, y_{\mathrm{n}}$ from a population whose main parameter is $\theta$, when the null hypothesis $H_{0}: \theta_{1}=\theta_{2}=\ldots=\theta_{n}$ is tested, it is named the homogeneity test. This suggests that heterogeneity is really all about a shifting population. This creates more confusion. The source of such confusion with respect to heterogeneity emanates from its ill-communication. It is evident that there is a lack of a clear definition of heterogeneity given by Hunink et al (Chapter 12), ${ }^{8}$ for details. Neither the Encyclopedia of Statistical Sciences nor the Encyclopedia of Biostatistics has even an entry as if it is not pertinent in statistical disciplines.

One comes across different types of data in scientific studies. Drawing data from a binomial population is one of them and the data should possess an under dispersion (ie, variance of the binomial distribution is smaller than its mean). ${ }^{1}$ From a Poisson population, the drawn random sample ought to reflect equality between the mean and variance. When the main (incidence rate) parameter of a Poisson chance mechanism is stochastically transient, the unconditional observation of the random variable convolutes to an inverse binomial model. ${ }^{9}$ The inverse binomial distribution is known to attest that the variance is larger than its mean (Stuart and $\operatorname{Ord}^{10}$ for details). Consequently, a comparison between the mean and variance characterizes only which type of binomial, Poisson, or inverse binomial possesses the underlying chance mechanism we are sampling from but does not inform anything about heterogeneity. Recently, Hassen et $\mathrm{al}^{11}$ employed a statistical concept behind a stochastic hybrid Susceptible-Infectious-Removed (SIR) framework in a Poisson chance mechanism for COVID-19 evolution and transmission in the Maghreb Central Regions (which consists of Tunisia, Algeria, and Morocco) in Western Africa. The COVID-19 pandemic is virulent and rapidly spreading in Maghreb Central Regions as much as elsewhere in other parts of the world. Their version of the SIR-Poisson model successfully predicted the range of the future infected cases since its emergence until the end of the confinement period April 2020. They estimated an average number of two contacts in Tunisia, while it was three contacts in Algeria and Morocco by an infected individual. According to them, the pandemics declined in each of the three countries but did not end. Furthermore, they detected an evolutionary change in the sense that the pandemics spreaded more rapidly in Morocco and Algeria than in Tunisia, though the most affected country was Algeria with more deaths despite a high number of cures.

With details about the probabilistic patterns among coronavirus confirmed, recovered or cured people and those that succumb as fatalities/deaths in the 32 states/ territories of India are given by Shanmugam. ${ }^{13}$ To track the confusion with respect to heterogeneity, let us consider the data given in Table $1,{ }^{12}$ describing the spread of COVID-19 among the voyagers in a Diamond Princess cruise ship, during the month of February 2020. The random variables $Y_{1}, Y_{2}$, and $Y_{3}$ denote, respectively, the number of COVID-19 cases, the number of asymptomatic cases and the number of symptomatic cases among them in time (date). Under a given COVID-19's prevalence rate, $\lambda>0$, the number $Y_{1}$ perhaps follows a Poisson probability pattern. For a given number of COVID-19 cases in a date, the number $Y_{2}$ perhaps follows a binomial probability pattern with parameters $\left(y_{1}, p\right)$, where $0<p<1$ denotes the chance for a COVID-19 case to exhibit no symptom. Naturally, the number $Y_{3}$ should follow a binomial probability pattern with parameters $\left(y_{1}, 1-p\right)$. There is an implicitness between $Y_{2}$ and $Y_{3}$ in the sense that $Y_{2}+Y_{3}=Y_{1}$. There are three-time oriented groups of

Table I COVID-I9 in Cruise Ship, 2020, Mizumoto et al ${ }^{16}$

\begin{tabular}{|l|l|l|l|l|l|l|l|}
\hline Date & $\boldsymbol{Y}_{\mathbf{3}}$ & $\boldsymbol{Y}_{\mathbf{2}}$ & $\boldsymbol{Y}_{\mathbf{1}}$ & $\bar{\lambda}=\overline{\boldsymbol{y}}_{1}$ & $\boldsymbol{s}_{\lambda}^{2}=\boldsymbol{\operatorname { V a r } ( \boldsymbol { Y } _ { 1 } )}$ & $\boldsymbol{O R}_{\frac{1 \rightarrow 2}{0 \rightarrow 1}}$ & $\boldsymbol{O d d s}_{\boldsymbol{Y}_{1}}$ \\
\hline Feb 15-16, 2020 & 29,32 & 38,38 & 67,70 & 68.50 & 4.50 & 0.50 & $1.7 \mathrm{E}-30$ \\
Feb 17-18, 2020 & 29,23 & 70,65 & 99,88 & 93.50 & 60.50 & 0.50 & $2.4 \mathrm{E}-41$ \\
Feb 19-20, 2020 & $1 \mathrm{I}, 7$ & 68,6 & 79,13 & 46.00 & 21.78 & 0.50 & $1.0 \mathrm{E}-20$ \\
\hline
\end{tabular}


COVID-19 incidences in Table 1. There is an observable heterogeneity among the three groups. In such scenario, it occurs due to a non-observable (parametric) heterogeneity. In data analysis, it is important to distinguish observable versus non-observable heterogeneity. A literature search does not provide an answer to this question.

It is evident that the average of the COVID-19 cases is an estimate of the COVID-19's prevalence rate (ie, $\hat{\lambda}$ in Table 1). Their estimates impress that the prevalence rate is transient, not constant across every pair of two-day duration diads. The Poisson population from which the COVID-19 cases are drawn ought to have been dynamic, implying the existence of a Poisson heterogeneity. The heterogeneity can be defined and captured as follows. This is the theme and purpose in this research article.

Likewise, given that a fixed number, $y_{1}$ of COVID-19 cases has occurred, a part of them might be asymptomatic cases, $y_{2}$ and the remaining are symptomatic cases, $y_{3}$. That is, $y_{2}$ and $y_{3}$ are complementary but $y_{2}+y_{3}=y_{1}$. The heterogeneity in each of the two sub-binomial populations is captured in terms of the observation $y_{1}$. We next try to define each binomial heterogeneity and compute them. In other words, we point out that the binomial heterogeneity is different from the Poisson heterogeneity. We then quantify their differences. A literature search offers no help to prove either the existence or absence of binomial heterogeneity in the data for $y_{2}$ or $y_{3}$ in Table 1. Hence, we continue probing matters with respect to heterogeneity.

The concept of heterogeneity seems to have escaped the researchers' scrutiny for a long time. It is time well spent and worthwhile to revive an interest in the construct of heterogeneity and that is exactly what this article is trying to accomplish. Hence, we first define and construct an approach for the idea of heterogeneity. To be specific, we first discuss Poisson heterogeneity and then take up binomial heterogeneity. May be our research direction about heterogeneity is, perhaps, pioneering. However, we believe that our approach is easily extendable for many other similar methodological setups. We illustrate our definition and all derived expressions for heterogeneity using COVID-19's data pertaining to the Diamond Princess cruise ship, Yokohama, 2020 as displayed in Table 1.

\section{Poisson and Binomial Heterogeneities}

Applied scientists emphasize that heterogeneity is of paramount importance in extracting and interpreting data evidence. Many data analysts are convinced that an unrecognized heterogeneity leads to a biased inference. To begin with, let us define heterogeneity. It is a factor causing non-similarities. In such scenario, we ought to identify its sources. We contemplate that there are two sources for heterogeneity to exist. One source ought to be from the drawn random sample of observations: $y_{1}, y_{2}, \ldots \ldots, y_{n}$, which we recognize as observable heterogeneity. The sampling variability, $\operatorname{Var}\left[f\left(y_{1}, y_{2}, \ldots \ldots, y_{n} \mid \theta\right)\right]$ for a selected statistic $f\left(y_{1}, y_{2}, \ldots . ., y_{n} \mid \theta\right)$ would express the observable heterogeneity. Another source is manifested in a nonobservable parameter, $\theta$ of the chance mechanism, which we recognize as non-observable heterogeneity. We wonder whether a non-uniform stochastic pattern of $\theta$ be indicative of the non-observable homogeneity. If the chance mechanism perversely selects a probability density function (pdf) for $\theta$, how would it manifest itself to portray the nonobservable heterogeneity? Both observable and nonobservable heterogeneity together, ought to be involved to make any definition of heterogeneity complete. If so, how do we integrate them? Often, under/over-dispersion is confused to be heterogeneity. It seems that the over/under dispersion $^{4}$ is precipitated by heterogeneity but not the other way. It is not obvious or proven so far in the literature on whether the converse is true. We focus only on Poisson and binomial populations to address heterogeneity and these arguments can be repeated for other populations considering similar methods.

\section{Poisson Heterogeneity}

Recall that the random integer, $Y_{1}$ denoting the number of COVID-19 cases in a place (like the Diamond Princess cruise ship) at a time (like February 2020) is a Poisson random variable with a specified prevalence rate, $\lambda>0$. That is, the conditional probability of observing $y_{1}$ number of COVID-19 cases under a prevalence rate $\lambda>0$ is $\operatorname{Pr}\left[Y_{1}=y_{1} \mid \lambda\right]=e^{-\lambda} \lambda^{y_{1}} / y_{1} ! ; y_{1}=0,1,2, \ldots ; \lambda>0$ with its expected number $E\left[Y_{1} \mid \lambda\right]=\lambda \quad$ and variability $\operatorname{Var}\left[Y_{1} \mid \lambda\right]=E\left[Y_{1} \mid \lambda\right]$. The reader is referred to Rajan and Shanmugam $^{14}$ for detailed derivations of the Poisson mean and variance. The prevalence parameter $\lambda$ itself is crucial in our discussions. The Poisson variability cannot be heterogeneity because the expected value also changes when the variability changes due to their inter-relatedness. Realize that no two individuals on the ship are assumed to have the same level of susceptibility to the COVID-19 virus. It is reasonable to imagine that the prevalence levels follow a conjugate, stochastic gamma distribution. The socalled conjugate prior knowledge in the Bayesian 
framework smooths the statistical analytic process. It is known that the conjugate prior for the Poisson distribution is gamma, whose pdf is

$$
c(\lambda \mid \alpha, \beta) d \lambda=e^{-(\alpha \lambda)}(\alpha \lambda)^{\beta-1} d(\alpha \lambda) / \Gamma(\beta) ; \alpha>0 ; \beta>0
$$

with an average. $E(\lambda \mid \alpha, \beta)=\frac{\beta}{\alpha} \quad$ and variability $\operatorname{Var}(\lambda \mid \alpha, \beta)=E(\lambda \mid \alpha, \beta) / \alpha$, where the parameters $\alpha$ and $\beta$ are recognized as hyper-parameters. ${ }^{14}$ Note that the hyperparameter $\alpha>0$ causes the variability in the COVID-19's prevalence rate to fluctuate up or down and hence, you would anticipate the heterogeneity to involve the hyperparameter $\alpha$. But the question is how?

We assume that the probability of observing a nonnegative COVID-19 cases, $y_{1}$ is a Poisson under a stable sampling population $\operatorname{Pr}\left(Y_{1} \mid \lambda\right)$ with an expected number $E\left(Y_{1} \mid \lambda\right)=\lambda$ and a variability $\operatorname{Var}\left(Y_{1} \mid \lambda\right)=E\left(Y_{1} \mid \lambda\right)$. With replications, the observable heterogeneity should become estimable. That is to mention, the maximum likelihood estimate (MLE) of the COVID-19 prevalence rate is the average number, $\bar{y}_{1}$, of the observations. To discuss the non-observable heterogeneity, we need to integrate its conjugate prior $c(\lambda \mid \alpha, \beta)$ for the non-observable $\lambda$ with the likelihood $\operatorname{Pr}\left(Y_{1} \mid \lambda\right)$ and it results in an update and it is called posterior pdf for $\lambda$. The expressions for nonobservable heterogeneity, observable heterogeneity and other expressions are derived and used.

\section{Binomial Heterogeneity}

In this section, we explore heterogeneity for two subbinomial processes emanating from a Poisson process. The asymptomatic number, $Y_{2}$ and symptomatic number, $Y_{3}$ of COVID-19 cases are two branching binomial random numbers out of the Poisson random number, $Y_{1}=$ $0,1,2, \ldots ;$ of COVID-19 cases. These two split random variables are complementary of each other in the sense that $Y_{2}+Y_{3}=Y_{1}$. Then, what are the underlying model for $Y_{2}$ and for $Y_{3}$ ? Are they correlated random variables? If so, what is their correlation? These are pursued in this section.

Let an indicator random variable, $I_{i}=1$ for a COVID19 person to show asymptomatic symptom with a probability, $0<p<1$ and $I_{i}=0$ to show symptomatic symptom with a probability, $0<1-p<1$. Then, for a fixed $y_{1}$, the random variable, $Y_{2}=\sum_{i=1}^{Y_{1}} I_{i}$ follows a binomial probability distribution with parameters $\left(y_{1}, p\right)$. Likewise, for a fixed $y_{1}$, the random variable, $Y_{3}=y_{1}-Y_{2}$ follows a complementary binomial distribution with parameters $\left(y_{1}, 1-p\right)$. That is,

$$
\begin{aligned}
\operatorname{Pr}\left(Y_{2}=y_{2} \mid y_{1}, p\right) & =\left(\begin{array}{l}
y_{1} \\
y_{2}
\end{array}\right) p^{y_{2}}(1-p)^{y_{1}-y_{2}} ; y_{2} \\
& =0,1,2, \ldots \ldots, y_{1} ; 0<p<1
\end{aligned}
$$

and

$$
\begin{aligned}
\operatorname{Pr}\left(Y_{3}=y_{3} \mid y_{1}, p\right) & =\left(\begin{array}{l}
y_{1} \\
y_{3}
\end{array}\right)(1-p)^{y_{3}} p^{y_{1}-y_{3}} ; y_{3} \\
& =0,1,2, \ldots \ldots, y_{1} ; 0<1-p<1
\end{aligned}
$$

The expressions for non-observable heterogeneity, observable heterogeneity and other expressions are given in Appendix I.

\section{Tango Index}

Lastly, we develop the Tango index and its significance level over the time period. Tango ${ }^{15}$ proposed an index to detect disease clusters in grouped data. This index received considerable attention in the literature. Following the line of thinking in Tango ${ }^{15}$ we could next assess the MLEs of several entities we estimated and displayed in Tables $1-3$. There are three groups of duration. The group 1 consists of 15 th and 16th of February 2020. The group 2 encloses data for 17th and 18th of February 2020. Group 3 contains data of 19th and 20th of February 2020. Two independent contrasts among the three groups are feasible. In an arbitrary style, we select to compare group 1 with group 2 and then group 2 with group 3. For this purpose, we formulate a contrast matrix

$$
A_{3 \times 3}=\left(\begin{array}{ccc}
-1 & 0 & 1 \\
1 & -1 & 0 \\
0 & 1 & 0
\end{array}\right)
$$

where the third column of the matrix needs no explanation. The Tango's statistic $T=\underline{r}^{\prime} A \underline{r}$ follows a chi-squared distribution with $v=2$ degrees of freedom (df), where $\underline{r}_{1 x 3}^{\prime}$ is a row vector of the MLE of a chosen entity in our analytic results in Tables 1 or 2 or 3. For an example, let $\underline{r}^{\prime}=(68.50,93.50,46.00)$ for the MLE of the COVID-19 prevalence rate, $\lambda$ in the groups. Then, the Tango's test statistic is $T=422.25$ with $v=2 \mathrm{df}$ and $p$-value $=2.03975 E-92$. Likewise, the Tango's test statistic value and its $\mathrm{p}$-value are calculated and displayed in Table 4 for other entities. 
Table 2 Results for Mizumoto et al's COVID-19 Data in Diamond Princess

\begin{tabular}{|l|c|l|l|l|l|l|}
\hline Date & $\boldsymbol{O R}_{\frac{Y_{3}}{Y_{2}}}$ & $\hat{\boldsymbol{H}}_{\boldsymbol{y}_{I}}$ & $\hat{\beta}$ & $\hat{\alpha}$ & $\boldsymbol{d}\left(\boldsymbol{y}_{1}, \lambda\right)$ \\
\hline $15,16 \mathrm{Feb} 2020$ & 943.88 & $0.27^{*}$ & 15.22 & 1042.72 & 857.81 & $\boldsymbol{H}_{\hat{\lambda}}$ \\
$17,18 \mathrm{Feb} 2020$ & $7.36 \mathrm{E}+17$ & $0.70^{*}$ & 1.54 & 144.50 & 0.93 \\
$19,20 \mathrm{Feb} 2020$ & $9.69 \mathrm{E}+\mathrm{II}$ & $0.65 *$ & 2.11 & 97.15 & 23.79 & 0.61 \\
\hline
\end{tabular}

Notes: *The observed level of Poisson heterogeneity was only 0.27 (a low amount) on February 15-16, 2020 but increased to 0.70 (a large amount) on February 17-18, 2020 and slightly decreased to 0.65 on February 19-20, 2020. It suggests that the chance-oriented Poisson mechanism was dynamic over the period.

Table 3 Results for Asymptomatic COVID-19 Cases in Mizumoto et al ${ }^{16}$

\begin{tabular}{|l|l|l|l|l|l|l|}
\hline Date & $\boldsymbol{1}-\overline{\boldsymbol{p}}=1-\boldsymbol{A v e}\left(\frac{\boldsymbol{y}_{2}}{\boldsymbol{y}_{1}}\right)$ & $\boldsymbol{s}_{\boldsymbol{p}}{ }^{2}=\boldsymbol{V} \hat{\boldsymbol{a}} \boldsymbol{r}\left(\frac{\boldsymbol{y}_{2}}{\boldsymbol{y}_{1}}\right)$ & $\hat{\boldsymbol{H}}_{\boldsymbol{y}_{1}, \gamma, \delta}$ & $\hat{\boldsymbol{H}}_{\boldsymbol{y}_{2} \mid \boldsymbol{y}_{1}}$ & $\boldsymbol{d}\left(\boldsymbol{y}_{2}, p\right)$ & $\boldsymbol{d}\left(\boldsymbol{Y}_{2}, \boldsymbol{Y}_{3}\right)$ \\
\hline 15, 16 Feb 2020 & 0.45 & 0.0002 & 0.99 & 0.95 & 37.12 & 6.85 \\
17, 18 Feb 2020 & 0.28 & 0.0004 & 0.98 & 0.89 & 66.60 & 41.14 \\
19, 20 Feb 2020 & 0.34 & 0.0796 & 0.10 & 0.74 & 29.70 & 14.72 \\
\hline
\end{tabular}

Table 4 Tango's Test Statistic** and Its P-value for Several Entities

\begin{tabular}{|l|l|l|l|l|l|l|l|l|}
\hline Tango Statistic & $\hat{\boldsymbol{H}}_{\boldsymbol{y}_{1}}$ & $\boldsymbol{H}_{\hat{\lambda}}$ & $\overline{\boldsymbol{p}}$ & $\hat{\boldsymbol{H}}_{\boldsymbol{y}_{1}, \gamma, \delta}$ & $\hat{\boldsymbol{H}}_{\boldsymbol{y}_{2} \mid \boldsymbol{y}_{1}}$ & $\boldsymbol{d}\left(\boldsymbol{y}_{1}, \lambda\right)$ & $\boldsymbol{d}\left(\boldsymbol{y}_{2}, p\right)$ & $\boldsymbol{d}\left(\boldsymbol{Y}_{2}, \boldsymbol{Y}_{3}\right)$ \\
\hline T with 2 df & 0.25 & 0.36 & $0.4 \mathrm{I}$ & 0.77 & $0.5 \mathrm{I}$ & 699420 & 260.66 & 751.20 \\
P-value & 0.87 & 0.83 & $0.8 \mathrm{I}$ & 0.67 & 0.77 & $0.0 \mathrm{E} / 00$ & $2.4 \mathrm{E}-57$ & $7.5 \mathrm{E}-164$ \\
\hline
\end{tabular}

Notes: **Recall that there are two types of heterogeneity. One is observable and the other is non-observable. The non-observable heterogeneities $H_{\hat{\lambda}}$ of the Poisson count, $y_{1}$ and of the non-observable heterogeneity, $\hat{H}_{y_{1}, \gamma, \delta}$ of the binomial count, $y_{2}$ are high on February I5-18, 2020 but decreased later on February 19-20, 2020 (Figure 2 for non-observable and Figure 3 for observable). The above-mentioned behavior of the non-observable heterogeneities are confirmed by the Tango's indices also. The isomorphic relationship (that is factor, $e^{-(1-p) \lambda}$ in expression (9) to visualize their interplay) between the parameters $p$ and $\lambda$ is illustrated in Figure 4 .

\section{Illustrating Using COVID-I 9 Data of the Diamond Princess Cruise Ship}

In this section, we illustrate all the concepts and expressions of Poisson and Binomial Heterogeneities. Let us consider the COVID-19 data in Table 1 for the Diamond Princess Cruise Ship, 2020. The Diamond Princess is a cruise ship registered in Britain and operated across the globe. During a cruise that began on 20 January 2020, positive cases of COVID-19 linked to the pandemic were confirmed on the ship in February 2020. Over 700 people out of 3711 became infected (567 out of 2666 passengers and 145 out of 1045 crew), and 14 passengers died. To be specific, on the 15th of February 2020, 67 people were infected, on the 16th of February 2020, 70 people were infected, on the 17th of February 2020, there were 99 COVID-19 cases, on the 18th of February, another 88 cases were confirmed. The US government initially asked Japan to keep the passengers and crew members on board the ship for 14 days. The US government, however, changed its policy to bring them to Travis Air Force Base in California and the Base in San Antonio, Texas.

For each specified day in the first column in Table 1, the estimate of the COVID-19's prevalence rate and its variance are calculated using expressions $\hat{\lambda}=\bar{y}_{1}$ and $\operatorname{Va} r\left(Y_{1} \mid \lambda\right)=s_{y_{1}}^{2}$. Both the prevalence and its variability increased and then decreased over the days. However, their correlation, $\hat{\rho}_{Y_{2}, Y_{3}}$ is calculated using the observed numbers on $y_{2}$ and $y_{3}$ for each day (see in Table 2) and the estimated correlations had been stable over the days. Substituting $\hat{\lambda}=\bar{y}_{1}$ and $\operatorname{Va} r\left(Y_{1} \mid \lambda\right)=s_{y_{1}}^{2}$ in the expression

$$
\hat{H}_{\lambda}=\frac{\hat{\lambda}}{\hat{\lambda}+\operatorname{Vâ} r\left(Y_{1} \mid \lambda\right)}
$$

we obtained the non-observable heterogeneity and displayed in Table 2. The non-observable Poisson heterogeneity for $y_{1}$ was high in the beginning day, came down later but only to increase later. Using $\hat{\lambda}=\bar{y}_{1}$ and $\operatorname{Var} r\left(Y_{1} \mid \lambda\right)=s_{y_{1}}^{2}$ in the expression 


$$
\hat{H}_{\bar{y}_{1}}=\left[1+\frac{\left(1+\frac{\bar{\lambda}}{s_{\lambda}^{2}}\right)}{6}\right]^{-1}
$$

we obtained the observable heterogeneity and displayed in Table 2. The observable Poisson heterogeneity was low in the beginning day, increases and then to decrease. Note in Table 2 that the observable and non-observable Poisson heterogeneities are inversely proportional. In other words, the estimate of the shape and scale parameter in the Bayesian approach are respectively $\hat{\alpha}=\frac{\bar{\lambda}^{2}}{s_{\lambda}^{2}}$ and $\hat{\beta}=\frac{\bar{\lambda}}{s_{\lambda}^{2}}$ (see their values in Table 2). The shape parameter value decreased consistently over the days. The scale parameter was high to begin with, then increased later. The distance, $d\left(y_{1}, \lambda\right)$ between the observable and non-observable Poisson mechanism for $y_{1}$ is calculated using the expression

$$
d\left(y_{1}, \lambda\right)=\{\beta(1-\beta) \pm 1\}\left(\frac{\alpha}{\{1+\beta\}^{2}}\right)
$$

and displayed in Table 2. Notice that the distance was large to begin with, decreased then but to increase later over the days.
Note that we compute $\hat{p}_{i}=\frac{y_{2}}{y_{1}}$ for the $i^{t h}$ day. Then, we calculate the average: $\bar{p}=\sum_{i=1}^{2} p_{i} / 2$ and the variance: $s_{p}^{2}=\frac{\left(p_{1}-p_{2}\right)^{2}}{4},(\hat{p}$ in Table 1$)$ and it had been steadily increasing over the days since 15 February 2020. This is something valuable for the medical professionals learning the clinical nature of COVID-19. Using the expression,

$$
\operatorname{odds}_{Y_{2}} \approx e^{-p \lambda}\left\{1+e^{-p(1-p) \lambda}\right\}
$$

in Binomial Heterogeneity, we calculated the odds for a COVID-19 case to become an asymptomatic type and displayed in Table 2.

Likewise, using the expression

$$
\operatorname{odd}_{Y_{3}} \approx e^{-(1-p) \lambda}\left\{1+e^{-p(1-p) \lambda}\right\}
$$

we estimated the odds for a COVID-19 case to become a symptomatic case and displayed in Table 2. Notice that both odds $\left(O d d s_{Y_{2}}\right.$ and $\left.O d d s_{Y_{3}}\right)$ are low but their odds ratio,

$$
O R_{\frac{Y_{3}}{Y_{2}}}=e^{-(1-2 p) \lambda}
$$

is not negligible but reveals that the situation is favorable to symptomatic rather than asymptomatic. This

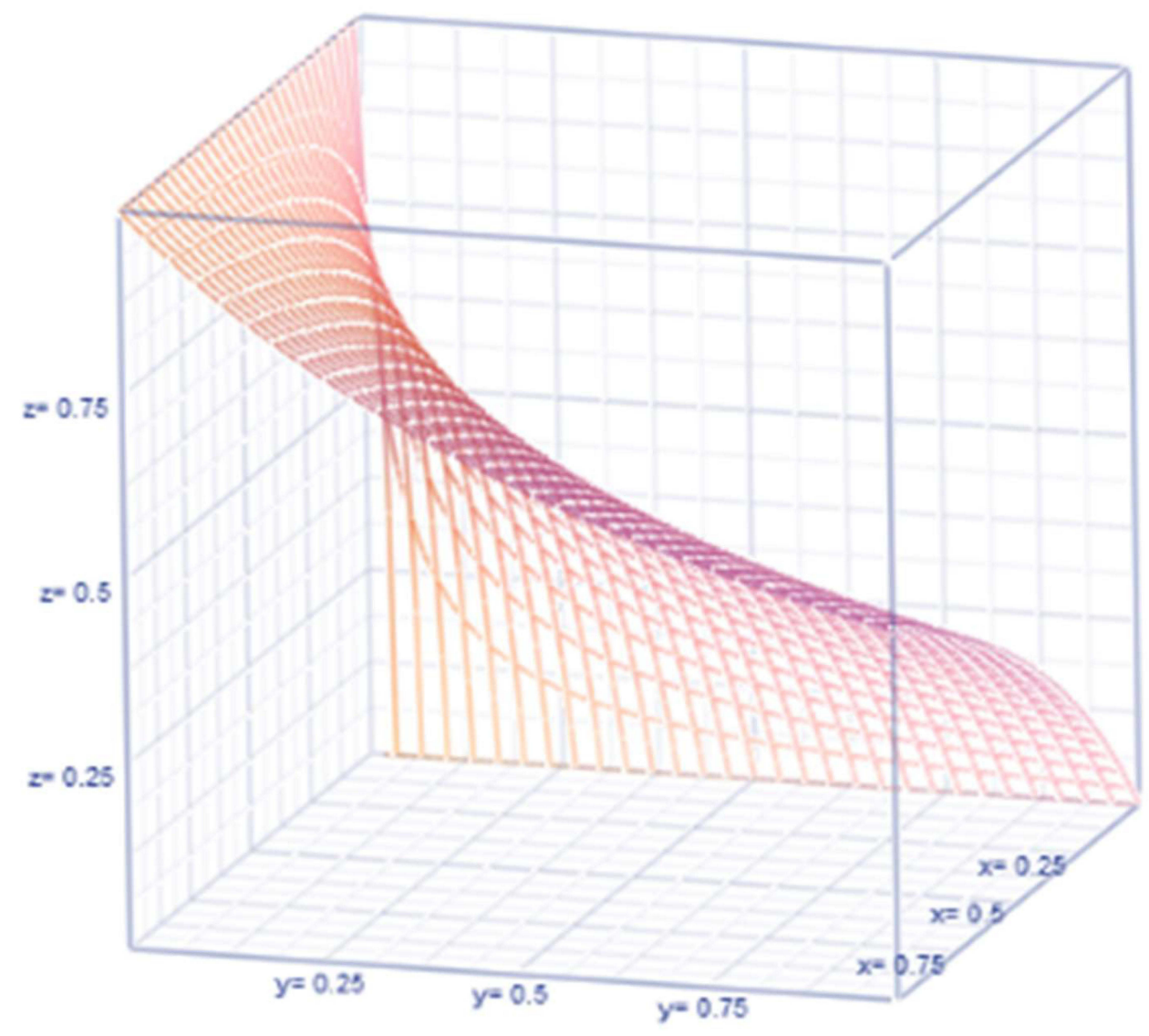

Figure I Distance, $d\left(y_{1}, \lambda\right)$ in Poisson. 


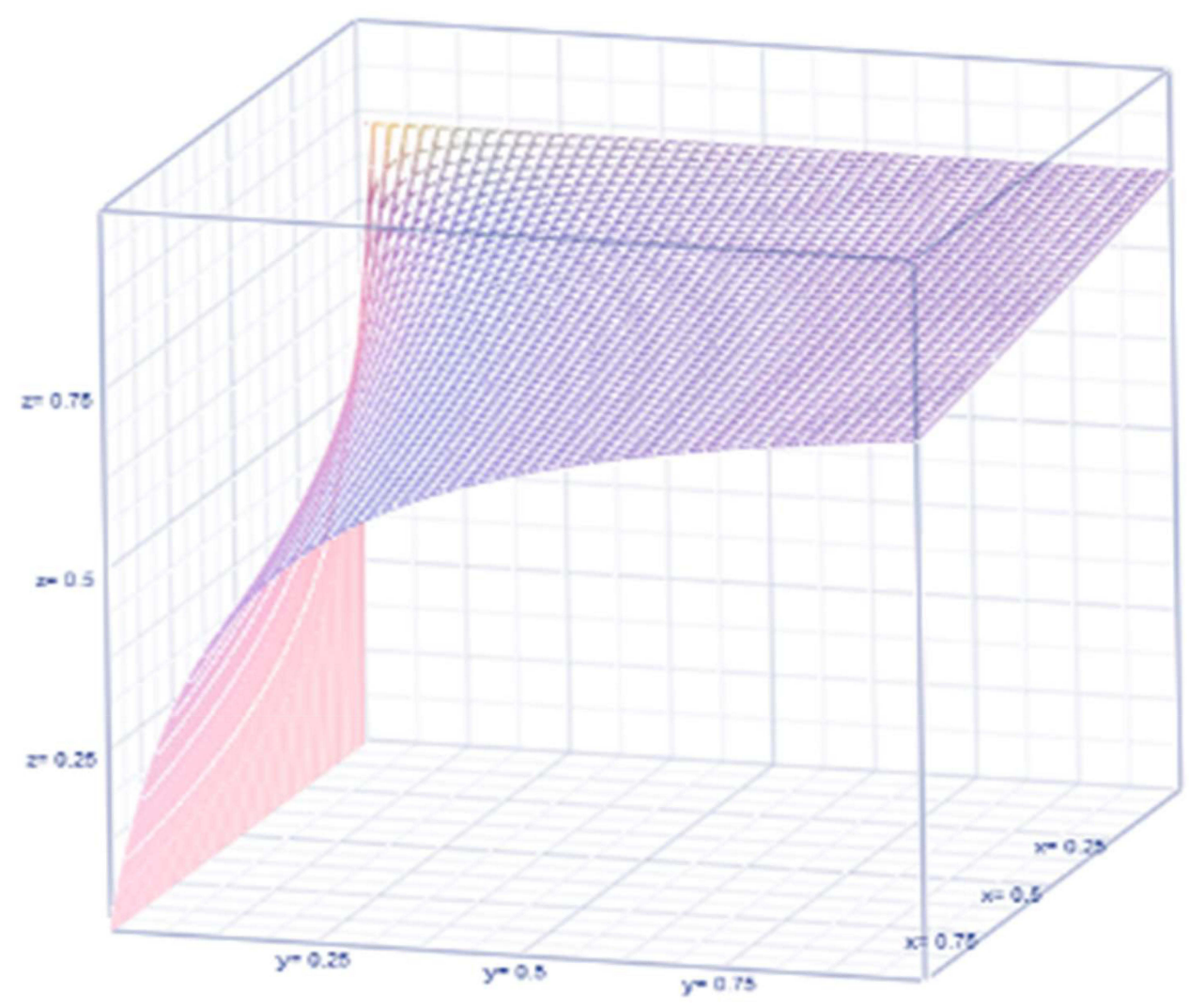

Figure 2 Non-observable heterogeneity.

discovery is feasible because of the approach, and it is an eye-opening reality for the medical professionals in their desire to control the spread of the COVID-19 virus. Both the observable, $\hat{H}_{y_{2} \mid y_{1}}$ and non-observable, $\hat{H}_{y_{1}, \gamma, \delta}$ binomial heterogeneity (see their values in Table 3) were decreasing for the number, $y_{2}$ of asymptomatic COVID-19 cases. The distance, $d\left(y_{2}, p\right)$ between the observable and non-observable for asymptomatic cases was moderate in the beginning, then increased, and then decreased over the next days (see their values in Table 3). However, the distance, $d\left(Y_{2}, Y_{3}\right)$ between the observable, $y_{2}$ of the asymptomatic cases and the observable, $y_{3}$ of the symptomatic cases was narrow, then wider, and then moderate over the days (their values in Table 3).

For a COVID-19 case to become a symptomatic type, the chance is moderate to less and then more over the days $(1-\bar{p}$ in Table 3$)$. The estimate of the shape and scale parameter happened to be $\hat{\gamma}$ and $\hat{\delta}$ respectively (see their values in Table 3 ). Both the shape parameter and the scale parameter values decreased drastically over the days. From the p-values in Table 4 , we infer that the prevalence rate, $\hat{\lambda}$, the distances, $d\left(y_{1}, \lambda\right), d\left(y_{2}, p\right)$ and $d\left(Y_{2}, Y_{3}\right)$ do differ significantly over the three groups of diad days (Figure 1). The chance for COVID-19 to become an asymptomatic type does not differ significantly across the three groups. On the contrary, the non-observable heterogeneities $H_{\hat{\lambda}}$ of the Poisson random number, $y_{1}$ and $\hat{H}_{y_{1}, \gamma, \delta}$ of the binomial random number, $y_{2}$ are not significant. Likewise, the observable heterogeneities $\hat{H}_{y_{1}}$ of the Poisson random number, $y_{1}$ and $\hat{H}_{y_{2} \mid y_{1}}$ of the binomial random number, $y_{2}$ for a given $y_{1}$ are not significant.

\section{Discussion and Conclusion}

The conclusions are reached based the new novel method that is generated in this article as there is no appropriate model or method in the literature. The sample size is sufficient. The control and experimental groups are not applicable in our discussion because of openly observed (not 


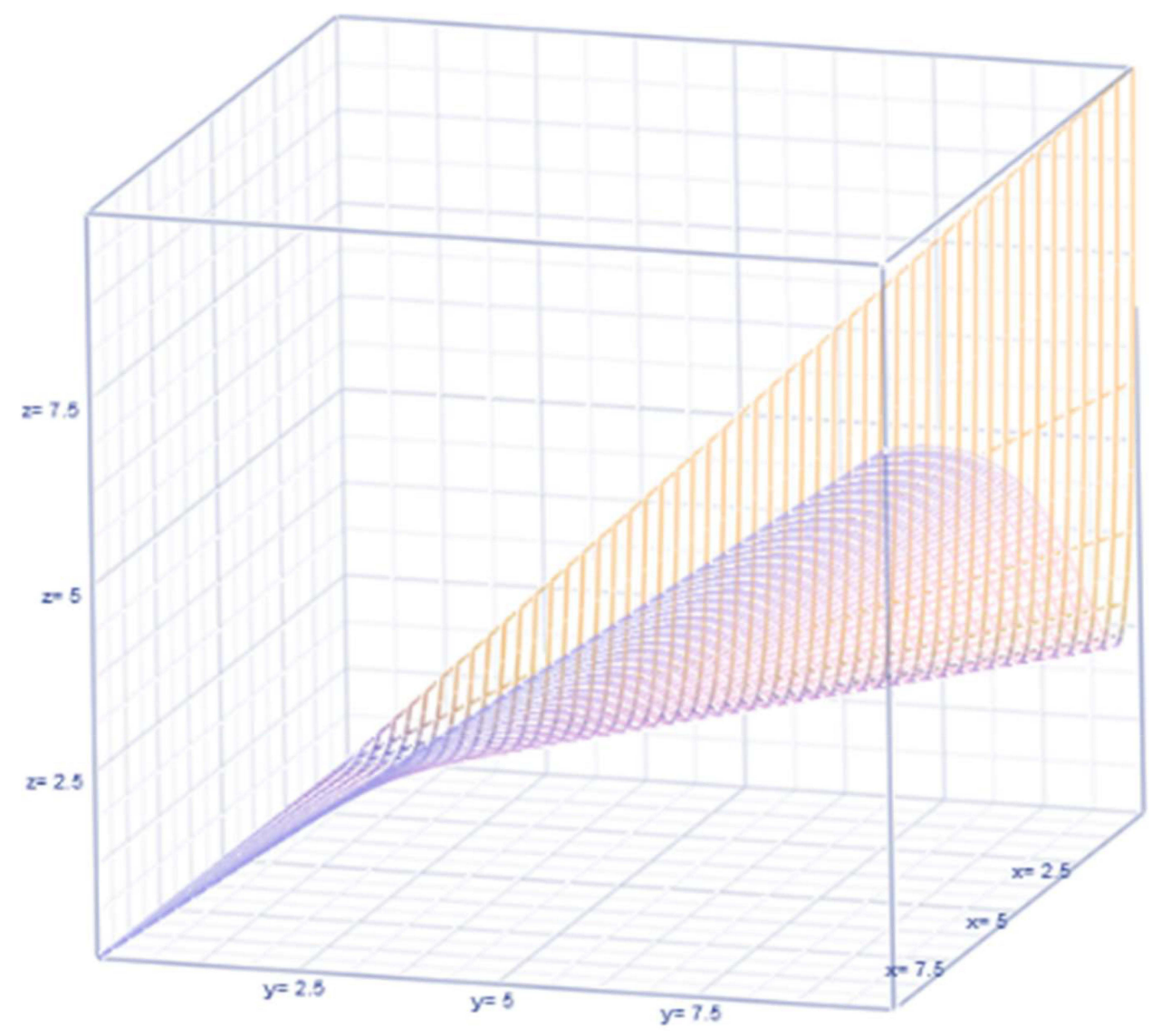

Figure 3 Observable heterogeneity.

experimental) collection of COVID-19. The risk ${ }^{3}$ of contracting the COVID-19 virus during a cruise is more than in a community setting as confined spaces discourage nonpharmaceutical mitigation strategies such as social distancing, to be weakly implemented and, not to mention, breathing air is tightly internalized. More nations are afraid to let the voyagers come ashore at the seaports. ${ }^{3}$ Not even the ships are permitted to dock at the port as to not complicate virus mitigation efforts by the local surrounding communities. The scenario seems to be anti-humanistic. Crew members are known to have committed suicide on the ship itself. The medical doctors and/or pharmaceutical service were strained due to the infected and COVID-19 free voyagers. Lack of clear symptoms among those that were infected added to difficulties in managing the COVID-19 crisis onboard the ship, and for any ship for that matter. Most importantly, how do we dispose of the COVID-19 fatalities (bodies), in a safe manner?

Amid uncertainties about the root cause and/or the appearance of any symptoms, the best modelers can do (as it is done in this article) is to devise a methodology to address the observable as well as non-observable heterogeneity, estimate the proportion of COVID-19 cases to be asymptomatic, estimate the odds of becoming symptomatic, and the odds ratio for asymptomatic in comparison to those symptomatic among COVID-19 cases (Figure 5). Some of these are non-trivial to the professional experts dealing with the intention of reducing the spread of COVID-19 if not its total control. Still much of COVID-19 is a mysterious pandemic. It is clear that non-pharmaceutical mitigation strategies such as social distancing, utilization of face coverings, frequent hand sanitization, infected people quarantine on board, and severely controlled ship cleanliness and sanitation standards are required; this may only be successful with limited numbers of passenger and crew members. Given the nature of the disease, its heterogeneity and human social norms, pre-voyage and post-voyage quick testing procedures may become the new standard for cruise ship passengers and crew. The technological advances in testing provided today would facilitate more humanistic treatment as compared to archaic quarantine and 


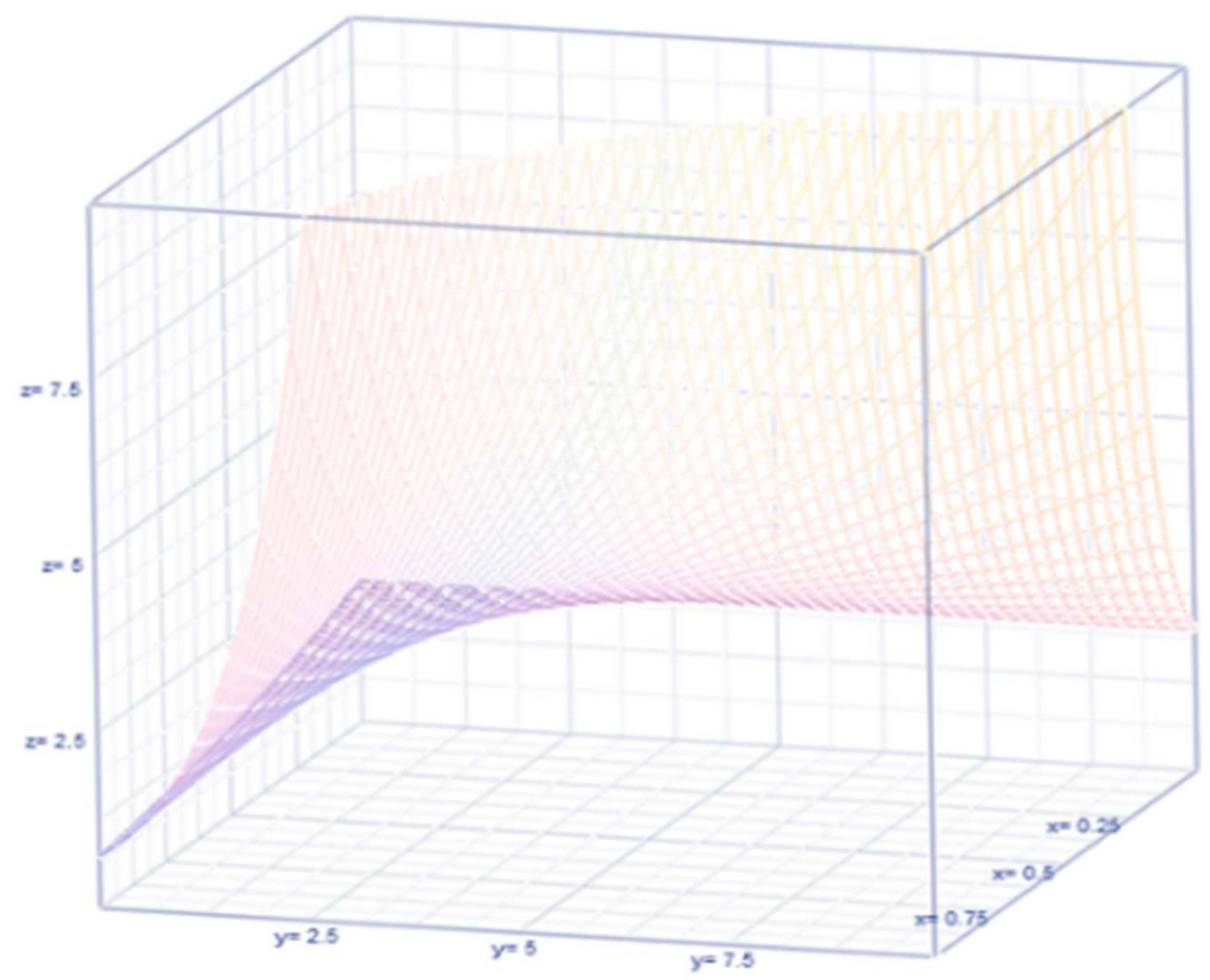

Figure 4 The configuration isomorphic factor $e^{-(1-2 p) \lambda}$.

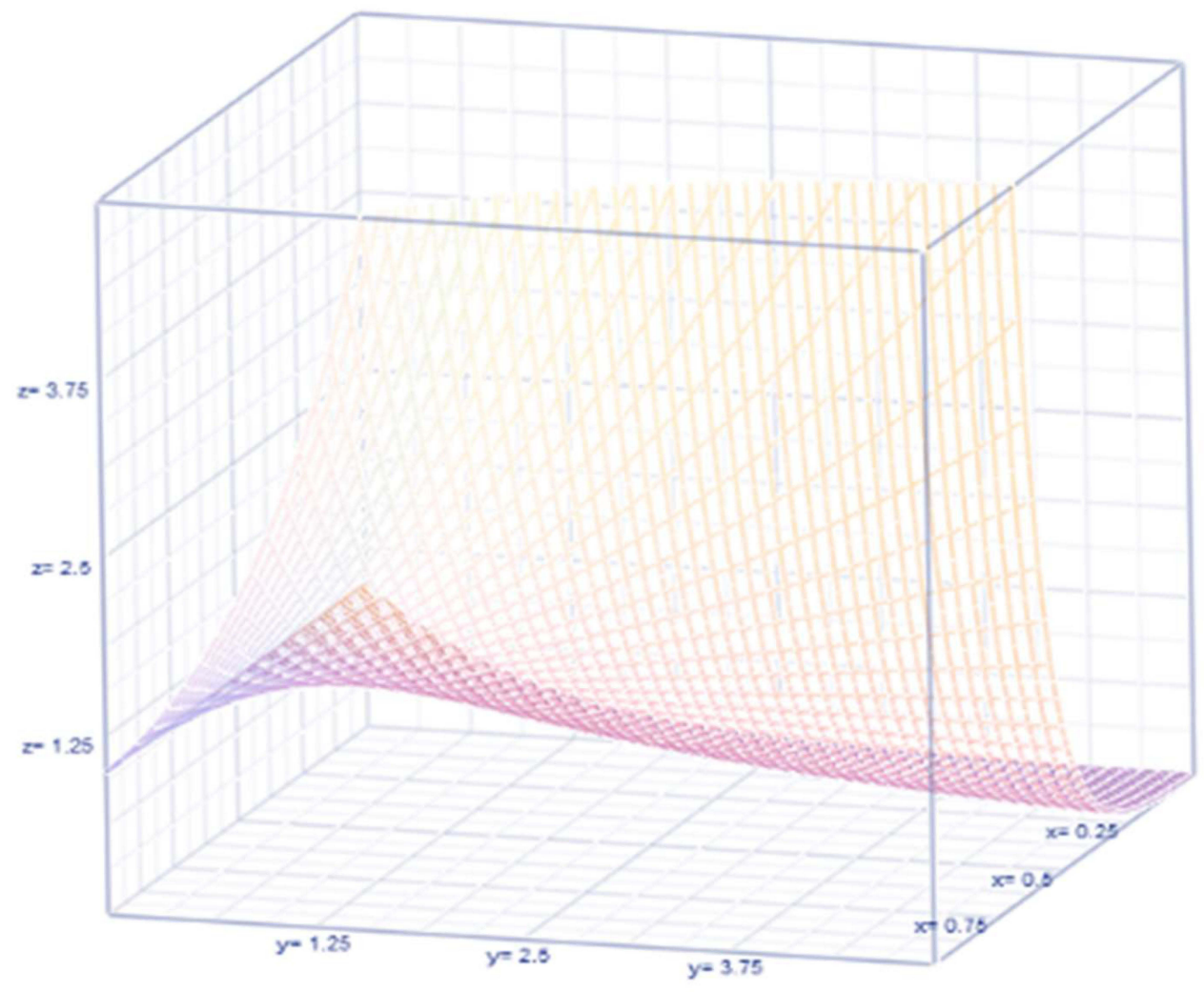

Figure 5 Odds for asymptotic. 
isolation practices for all onboard ship. With quick testing, identification of those infected and thus not allowed to embark on a cruise or quarantine those disembarking, and other mitigation strategies, the popular cruise adventure could be available safely again. Whatever the procedures implemented, the methodological purpose of this study should add valuable insight in the modeling of disease and specifically, the COVID-19 virus.

The novel method that is presented in this article offers an approach to extract the findings from any data in other disciplines also. The future research work ought to involve building data mining and/or regression fit for data with predictors on the pandemic incidences. The data collection methods could be survey or experimental design based as the method is then applicable to a variety of disciplines including marketing, sociology, business, computer information systems, communication engineering, medicine, public health, and artificial intelligence, among others.

\section{Data Sharing Statement}

There are no other data or materials other than what are in the manuscript itself.

\section{Acknowledgments}

The authors thanks Texas State University and The University of Texas at Tyler Health Science Center for the support to write this article.

\section{Funding}

There is no funding to report.

\section{Disclosure}

The authors report no conflicts of interest for this work.

\section{References}

1. Blumenfeld D. Operations Research Calculations Handbook. Boca Raton, Florida: CRC Press; 2020.

2. Elston R, Olson J, Palmer L. Biostatistical Genetics and Genetic Epidemiology. Baffins Lane, Chichester, West Sussex, UK: Wiley Press; 2002.

3. Khokhlov V. Conditional value-at-risk for elliptical distributions. Eur J Manag Bus Econ. 2016;2(6):70-79.

4. Shanmugam R, Radhakrishnan R. Incidence jump rate reveals over/ under dispersion in count data. Int J Data Anal Inf Syst. 2011;3(1):1-8.

5. Spreeuw J. Heterogeneity in hazard rates in insurance, Tinbergen Institute of Research Series, 210 [Ph. D. thesis] Amsterdam: University of Amsterdam; 1999.

6. Ecochard J. Heterogeneity in fecundability studies: issues and modeling. Stat Methods Med Res. 2006;15:141-160. doi:10.1191/ 0962280206sm436oa

7. Hope T, Norris P. Heterogeneity in the frequency distribution of crime victimization. J Quant Criminol. 2013;29(4):543-576. doi:10.1007/s10940-012-9190-x

8. Hunink MG, Weinstein MC, Wittenberg E, et al. Decision Making in Health and Medicine: Integrating Evidence and Values. Cambridge, UK: Cambridge University Press; 2018.

9. Ross S. A First Course in Probability. 6th ed. Upper Saddle River, New Jersey: Prentice Hall; 2002:07458.

10. Stuart A, Ord K. Kendall's Advanced Theory of Statistics, Volume 1. London: Oxford University Press; 2015.

11. Hassen HB, Elaoud A, Salah NB, et al. A SIR Poisson model for COVID-19 evolution and transmission inference in the Maghreb Central Regions. Arab J Sci Eng. 2021;46(1):93-102. doi:10.1007/ s13369-020-04792-0

12. Mizumoto K, Chowell G. Transmission potential of the novel coronavirus (COVID-19) onboard the diamond Princess Cruises Ship, 2020. Infect Dis Model. 2020;5:264-270. doi:10.1016/j.idm.2020. 02.003

13. Shanmugam R. Probabilistic patterns among coronavirus confirmed, cured and deaths in Thirty-two India's States/Territories. Int J Ecol Econ Stat. 2020;41:45-56.

14. Rajan C, Shanmugam R. Discrete Distributions in Engineering and the Applied Sciences, Synthesis Lectures on Mathematics and Statistics. Vol. 12. 82 Winter sport Ln, Williston, VT 05495, USA: Morgan \& Claypool Press; 2020:1-227.

15. Tango T. The detection of disease clustering in time. Biometrics. 1984;40:15-26. doi:10.2307/2530740

16. Mizumoto K, Kagaya K, Zarebski A, Chowell G. Estimating the asymptomatic proportion of coronavirus disease 2019 (COVID-19), cases on board the Diamond Princess cruise ship, Yokohama, 2020. Euro Surveill. 2020;25(10):1560-7917. doi:10.2807/1560-7917. ES.2020.25.10.2000180
Journal of Multidisciplinary Healthcare

\section{Publish your work in this journal}

The Journal of Multidisciplinary Healthcare is an international, peerreviewed open-access journal that aims to represent and publish research in healthcare areas delivered by practitioners of different disciplines. This includes studies and reviews conducted by multidisciplinary teams as well as research which evaluates the results or conduct of such teams or healthcare processes in general. The journal covers a very wide range of areas and welcomes submissions from practitioners at all levels, from all over the world. The manuscript management system is completely online and includes a very quick and fair peer-review system. Visit http://www.dovepress.com/testimonials. php to read real quotes from published authors. 